\title{
The Effect of Thermal Radiation on the Surface Catalytic Chemical Reaction ${ }^{* *}$
}

\section{GHEORGHE JUNCU*}

Politehnica University of Bucharest, Department of Chemical and Biochemical Engineering, 1 Polizu Str., 011061, Bucharest, Romania

Abstract. The effect of thermal radiation on the two - dimensional, steady-state, coupled heat and mass transfer from a fluid flow to a sphere in the presence of an exothermal catalytic chemical reaction on the surface of the sphere is investigated in the present work. The $P_{1}$ approximation models the radiative transfer. The finite difference method was used to discretize the mathematical model equations. The discrete equations were solved by the defect correction - multigrid method. The influence of thermal radiation on the sphere surface temperature, concentration and reaction rate was analysed. It was found that, for high values of the radiation conduction parameter, thermal radiation has a significant effect on the surface reaction.

Keywords: heat transfer, mass transfer, convection-radiation; surface reaction, $P_{1}$ approximation, finite difference

\section{Introduction}

Many problems with broad applications in aerothermochemistry, environmental, industrial and lifescience processes are mathematically modeled by convection - diffusion equations with surface chemical reactions. A review of the earlier articles published on this topic was made by Chung [1]. In the framework of the boundary layer formalism, the convection - diffusion surface chemical reaction problem was analysed by (due to the aim of this work the citation is restricted to external flow problems):

- $\quad$ flat plate geometry - [2-12];

- $\quad$ spherical geometry - [13-17].

It must be mentioned the followings: (1) the multiplicity of the steady states was investigated by Lindberg and Schmitz [2,13] and Mihail and Teodorescu [5]; (2) the case of a non-Newtonian fluid flow was analysed by Mihail [4] and Elperin et al. [12]; (3) Merkin studied the case of homogeneousheterogeneous isothermal chemical reaction.

The coupled heat and mass transfer to a sphere with a first-order, non-isothermal chemical reaction on the surface of the sphere was analysed numerically by Juncu and Mihail [18] without the boundary layer assumptions. Paterson [19] investigated the case of isothermal surface reaction in spherical geometry using the film model for mass transfer.

The aim of the present work is to make a first step in the investigation of the effect of thermal radiation on the forced convection heat and mass transfer to a sphere in the presence of an exothermal catalytic surface chemical reaction. The flow past the sphere is considered steady, laminar and incompressible. To the best of our knowledge, this problem is reported for the first time here. The $P_{1}$ approximation $[20,21]$ was used to model the radiative transfer. In spite of its simplicity, the $P_{1}$ approximation is the best compromise between accuracy and computational efficiency.

This paper is organized as follows. The next section describes the mathematical model of the problem. Section Method of solution presents the numerical algorithm. The numerical experiments

\footnotetext{
*email: juncu.gheorghe@yahoo.com, juncu@cael.pub.ro

**This article is dedicated to the birth's centenary of professor Raul Mihail, the founder of the Romanian school of chemical and biochemical reactors.
} 
made and the results obtained are presented in section Results. The concluding remarks of this work are briefly mentioned in the final section.

\section{Mathematical model}

Let us consider a sphere placed in a laminar, incompressible, steady flow of a Newtonian fluid. The flow configuration and coordinate system are shown in Figure 1. The diameter of the sphere $d$ is assumed considerably larger than the mean free path of the surrounding fluid. The free stream velocity, temperature and concentration are $U_{\infty}, T_{\infty}$ and $C_{\infty}$. On the surface of the sphere an exothermic, first-order catalytic chemical reaction occurs. The physical properties are constant and isotropic. The effects of buoyancy, viscous dissipation and work done by pressure forces are considered negligible. The fluid is assumed to be a gray, emitting, absorbing and isotropic scattering medium.

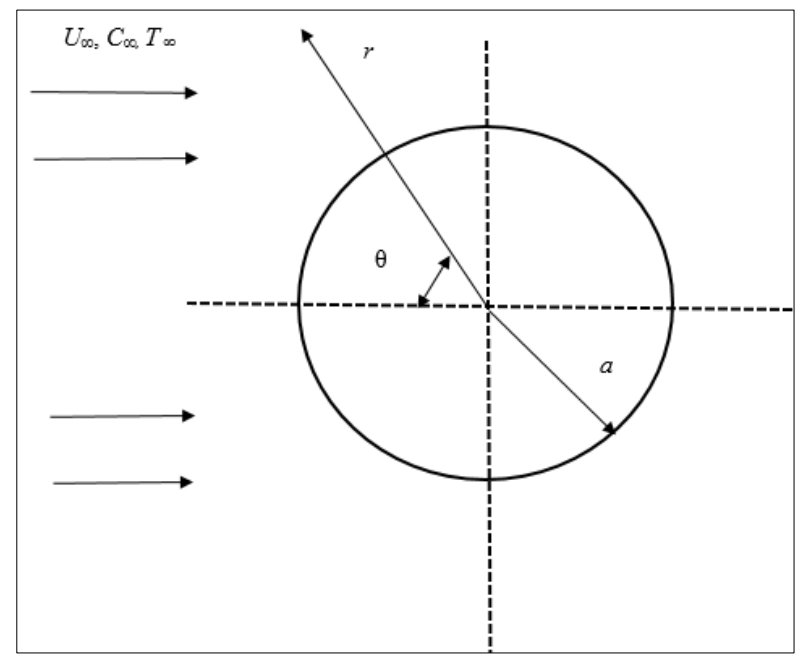

Figure 1. Schematic of the problem

For the assumptions discussed previously, the dimensionless energy and chemical species balance equations for a participating medium (the radius of the sphere $a$ is considered the length scale and the free stream velocity $U_{\infty}$ the velocity scale), expressed in dimensionless spherical coordinate system $(r$, $\theta)$, are:

-Chemical species balance equation

$$
\frac{\operatorname{ReSC}}{2}\left(V_{R} \frac{\partial Y}{\partial r}+\frac{V_{\theta}}{r} \frac{\partial Y}{\partial \theta}\right)=\frac{1}{r^{2}} \frac{\partial}{\partial r}\left(r^{2} \frac{\partial Y}{\partial r}\right)+\frac{1}{r^{2} \sin \theta} \frac{\partial}{\partial \theta}\left(\sin \theta \frac{\partial Y}{\partial \theta}\right)
$$

-Energy balance equation

$$
\frac{R e P r}{2}\left(V_{R} \frac{\partial Z}{\partial r}+\frac{V_{\theta}}{r} \frac{\partial Z}{\partial \theta}\right)=\frac{1}{r^{2}} \frac{\partial}{\partial r}\left(r^{2} \frac{\partial Z}{\partial r}-r^{2} q_{r, r}\right)+\frac{1}{r^{2} \sin \theta} \frac{\partial}{\partial \theta}\left(\sin \theta \frac{\partial Z}{\partial \theta}-\sin \theta q_{r, \theta}\right)
$$

where $q_{r, r}$ and $q_{r, \theta}$ are the dimensionless normal and tangential components of the radiative heat flux vector $q_{r}, q_{r}=q_{r}\left(q_{r, r}, q_{r, \theta}\right)$. In the energy balance equation (2), the effect of thermal radiation is quantified by the divergence of the radiative heat flux vector $q_{r}$. The relation for the computation of the radiative heat flux depends on the mathematical model used for the radiative transfer.

The boundary conditions to be satisfied by the dimensionless concentration and temperature are:

-Symmetry axis $(\theta=0, \pi)$;

-Surface of the sphere $(r=1)$;

$$
\frac{\partial Z}{\partial \theta}=0, \frac{\partial Y}{\partial \theta}=0
$$




$$
\begin{gathered}
\frac{\partial Z}{\partial r}-q_{r, r}=-B \varphi^{2} Y \exp \left[\gamma\left(1-\frac{1}{Z}\right)\right] \\
\frac{\partial Y}{\partial r}=\varphi^{2} Y \exp \left[\gamma\left(1-\frac{1}{Z}\right)\right]
\end{gathered}
$$

-Free stream $(r \rightarrow \infty)$;

$$
Z=1.0, Y=1.0
$$

For $P_{1}$ approximation [21], the dimensionless radiative heat flux vector $q_{r}\left(q_{r, r}, q_{r, \theta}\right)$ satisfies the equation

$$
\frac{1}{r^{2}} \frac{\partial}{\partial r}\left(r^{2} q_{r, r}\right)+\frac{1}{r^{2} \sin \theta} \frac{\partial}{\partial \theta}\left(\sin \theta \frac{\partial q_{r, \theta}}{\partial \theta}\right)=-K^{2} R d\left(Z^{4}-G\right)
$$

where $G$ is the dimensionless directed - integrated intensity of the radiation, $K=k_{a} a, R d=$ $4 \sigma n^{2} T_{\infty}^{3} /\left(k_{a} \lambda\right)$. Substituting equation (4) into equation (2), it results:

$$
\frac{R e P r}{2}\left(V_{R} \frac{\partial Z}{\partial r}+\frac{V_{\theta}}{r} \frac{\partial Z}{\partial \theta}\right)=\frac{1}{r^{2}} \frac{\partial}{\partial r}\left(r^{2} \frac{\partial Z}{\partial r}\right)+\frac{1}{r^{2} \sin \theta} \frac{\partial}{\partial \theta}\left(\sin \theta \frac{\partial Z}{\partial \theta}\right)-K^{2} R d\left(Z^{4}-G\right)
$$

The dimensionless directed - integrated intensity of radiation $G$ verifies the equation [21]:

$$
\frac{1}{r^{2}} \frac{\partial}{\partial r}\left(r^{2} \frac{\partial G}{\partial r}\right)+\frac{1}{r^{2} \sin \theta} \frac{\partial}{\partial \theta}\left(\sin \theta \frac{\partial G}{\partial \theta}\right)-\mathfrak{B} K\left(G-Z^{4}\right)=0
$$

where $\mathfrak{B}=3 \beta$ a.

The boundary conditions to be satisfied by $G$ are [21]:

- $\quad$ Symmetry axis $(\theta=0, \pi)$;

- $\quad$ Surface of the sphere $(r=1)$;

- Free stream $(r \rightarrow \infty)$;

$$
\frac{\partial G}{\partial \theta}=0
$$

$$
-\frac{\partial G}{\partial r}+\mathfrak{B} \mathcal{E}\left(G-Z^{4}\right)=0
$$

$$
G=Z^{4}=1.0
$$

where $\mathcal{E}=\frac{\varepsilon}{2(2-\varepsilon)}$. The boundary condition (7c) considers radiative equilibrium at free stream. It must be mentioned that for the $P_{1}$ approximation, the dimensionless radiative heat flux is given by [21],

$$
q_{r}=-1 / \mathfrak{B} \operatorname{grad} G
$$

The physical quantities of interest are the sphere surface dimensionless average temperature and concentration $\bar{Z}_{S}, \bar{Y}_{S}$, the external effectiveness factor $\eta_{e}$, the local Nusselt / Sherwood number, $N u_{\theta} / S h_{\theta}$, and the average Nusselt / Sherwood number, Nu/Sh. The sphere surface dimensionless average temperature and concentration $\bar{Z}_{s}, \bar{Y}_{s}$, were computed with the relations:

$$
\begin{aligned}
& \bar{Z}_{s}=\left.\frac{1}{2} \int_{0}^{\pi} Z\right|_{r=1} \sin \theta d \theta \\
& \bar{Y}_{s}=\left.\frac{1}{2} \int_{0}^{\pi} Y\right|_{r=1} \sin \theta d \theta
\end{aligned}
$$

The external effectiveness factor was calculated with the relation: 


$$
\eta_{e}=\left.\frac{1}{2} \int_{0}^{\pi} Y\right|_{r=1} \exp \left[\gamma\left(1-\frac{1}{Z}\right)\right] \sin \theta d \theta
$$

Considering as driving force the difference $\left(\bar{Z}_{s}-1\right) /\left(1-\bar{Y}_{S}\right)$, the local Nusselt / Sherwood number based on the diameter of the sphere is given by:

$$
N u_{\theta}=-\left.\frac{2}{\overline{Z_{S}}-1} \frac{\partial Z}{\partial r}\right|_{r=1}+2 \frac{R d K \mathcal{E}}{\overline{Z_{S}}-1}\left(Z^{4}-G\right), S h_{\theta}=\left.\frac{2}{1-\bar{Y}_{S}} \frac{\partial Y}{\partial r}\right|_{r=1}
$$

The average $N u$ / Sh number was computed with the relation:

$$
N u=\frac{1}{2} \int_{0}^{\pi} N u_{\theta} \sin \theta d \theta, S h=\frac{1}{2} \int_{0}^{\pi} S h_{\theta} \sin \theta d \theta
$$

\section{Method of solution}

The mathematical model equations were solved numerically with the nested multigrid defectcorrection method [22]. The mass and energy balance equations $(1,5)$ were discretized with the upwind and centered finite difference schemes (a double discretization required by the defect - correction iteration) on a vertex-centered grid, [23]. The spatial derivatives of the radiative transfer equation (6) were approximated by the centered finite difference scheme. Numerical experiments were made on meshes with the discretization steps, $\Delta \theta=\pi / 64, \Delta r=1 / 64, \Delta \theta=\pi / 128, \Delta r=1 / 128$ and $\Delta \theta=\pi /$ $256, \Delta r=1 / 256$. The external boundary conditions (3d) and (7c) are assumed to be valid at a large but finite distance from the center of the sphere.

The defect - correction iteration was applied only to the discrete approximation of the mass and heat balance equations. Two multigrid cycles were used inside the defect - correction iteration step. The structure of the MG cycle is: 1) cycle of type V; 2) smoothing by alternating line Gauss Seidel method; 3) two smoothing steps are performed before the coarse grid correction and one after; 4) prolongation by bilinear interpolation for corrections; 5) restriction of residuals by full weighting. The radiative transfer equation was solved by the multigrid method previously presented. The velocity field $\left(V_{R}, V_{\theta}\right)$ were calculated solving numerically the Navier-Stokes equations. More information about the hydrodynamic computations can be viewed in [22].

The error criteria employed are: the discrete $L_{2}$ norm of the residuals and the discrete $L_{\infty}$ norm of the difference between the numerical solutions of two consecutive defect - correction iterations are smaller than $10^{-8}$. Results that can be used to validate the accuracy of the present computations are not available in literature. The mesh independence of the $\mathrm{Nu} / \mathrm{Sh}$ number and dimensionless sphere average surface temperature and concentration was the accuracy test used in the present computations.

\section{Results and discussions}

The dimensionless groups of the present problem can be divided into the following three classes: (1) convection - diffusion dimensionless groups, $\operatorname{Pr}, \operatorname{Re}$ and $S c$; (2) radiative dimensionless groups, $\mathfrak{B}, \mathcal{E}$, $K$ and $R d$; (3) surface reaction dimensionless groups $B, \varphi^{2}$ and $\gamma$.

The fluid flow past a rigid sphere is steady, laminar and axisymmetric for $R e \leq 210$, Johnson and Patel [24]. The numerical values considered for the $\operatorname{Pr}$ and Sc numbers are, $\operatorname{Pr}=1.0$ and $S c=2.0$. The values of the radiative dimensionless groups $\mathfrak{B}, \mathcal{E}$ and $K$ are given by the values of $a, k_{a}, \beta$ and $\varepsilon$. The values of $k_{a}$ and $\beta$ were taken from [21]. The emissivity $\varepsilon$ takes values in the range, $0.5 \leq \varepsilon \leq 0.9$. The values considered for the radiation - conduction parameters $R d$ are, $0 \leq R d \leq 10^{4}$. The numerical values assumed for the radius of the sphere are, $a \sim 0.01 \mathrm{~m}$, Wijngaarden et al. [25]. The values for the Prater number, $B$, the dimensionless activation energy, $\gamma$ and the dimensionless reaction parameter, $\varphi^{2}$, were selected following the recommendations made by Aris [26] and Froment et al. [27].

The main aspect analysed in this section is the influence of the radiation dimensionless groups on the surface catalytic chemical reaction. The values of the reaction parameters were chosen such that, when 
thermal radiation is neglected, the consumption of the reactant on the surface of the sphere is almost complete.

The influence of the radiation - conduction dimensionless group $R d$ on the sphere surface average dimensionless concentration, $\bar{Y}_{s}$, and temperature, $\bar{Z}_{s}$, is presented in Figures 2 and 3, respectively. The three curves plotted in each figure were computed for three different values of the sphere radius. Figures 2 and 3 show that the increase in $R d$ increases $\bar{Y}_{s}$ and decreases $\bar{Z}_{s}$. The variation of $\bar{Y}_{s}$ and $\bar{Z}_{s}$ versus $R d$ is monotonous but not smooth. It is similar to a step function. The decrease in the sphere radius increases the $R d$ value for which the jump occurs. One can state that the ignition / extinction of the surface catalytic chemical reaction also depends on the intensity of the radiative transfer. In the next paragraphs, the $R d$ values for which $\bar{Y}_{S}$ and $\bar{Z}_{S}$ vary abruptly will be named critical domain.
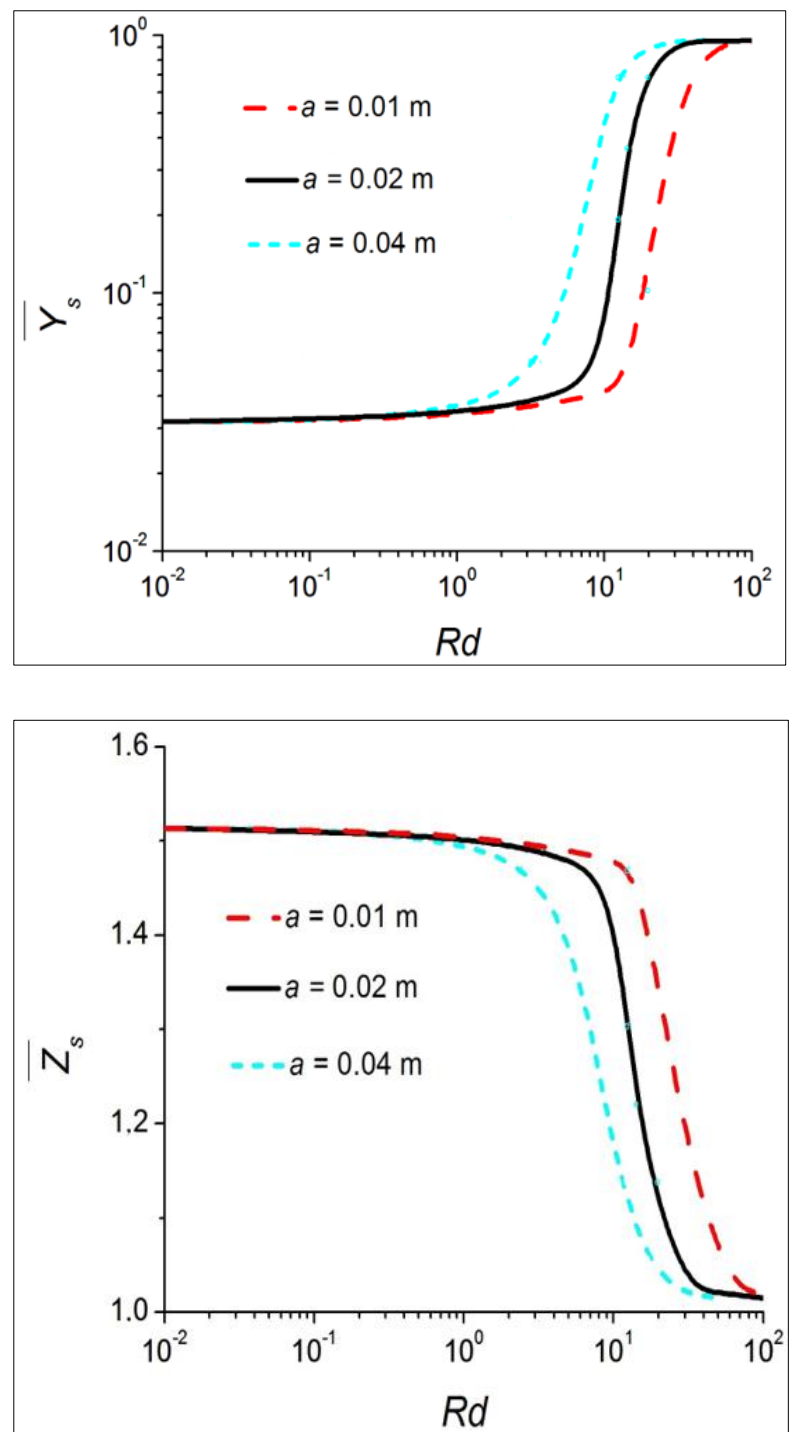

Figure 2. The influence of the radiation conduction parameter $R d$ on the surface average dimensionless concentration for $R e=100, B=0.43, \gamma=20.0, \varphi^{2}=0.16$, $\mathcal{E}=0.269\left(k_{a}=1 \mathrm{~m}^{-1}, \beta=3 \mathrm{~m}^{-1}\right.$ and $\left.\varepsilon=0.7\right)$
Figure 3. The influence of the radiation conduction parameter $R d$ on the surface average dimensionless temperature for $R e=100, B=0.43, \gamma=20.0, \varphi^{2}=0.16$ and $\mathcal{E}=0.269\left(k_{a}=1 \mathrm{~m}^{-1}, \beta=3 \mathrm{~m}^{-1}\right.$ and $\left.\varepsilon=0.7\right)$

The effect of the radiation - conduction parameter $R d$ on the dimensionless average heat and mass transfer rates and on the external effectiveness factor is presented in Figure 4. Figure 4 shows that outside the critical domain the influence of $R d$ on $N u, S h$ and $\eta_{e}$ is not significant. The increase in $R d$ increases slowly $N u$ and decreases slowly $\eta_{e}$ and $S h$. Inside the critical domain, $N u$ increases slowly but $S h$ decreases faster. The external effectiveness factor decreases abruptly inside the critical domain. The increase in $N u$ for values of $R d$ greater than the critical domain values is given by the decrease in the driving force and the increase in $R d$. The increase in $S h$ for values of $R d$ greater than the critical domain values is mainly given by the decrease in the driving force. 

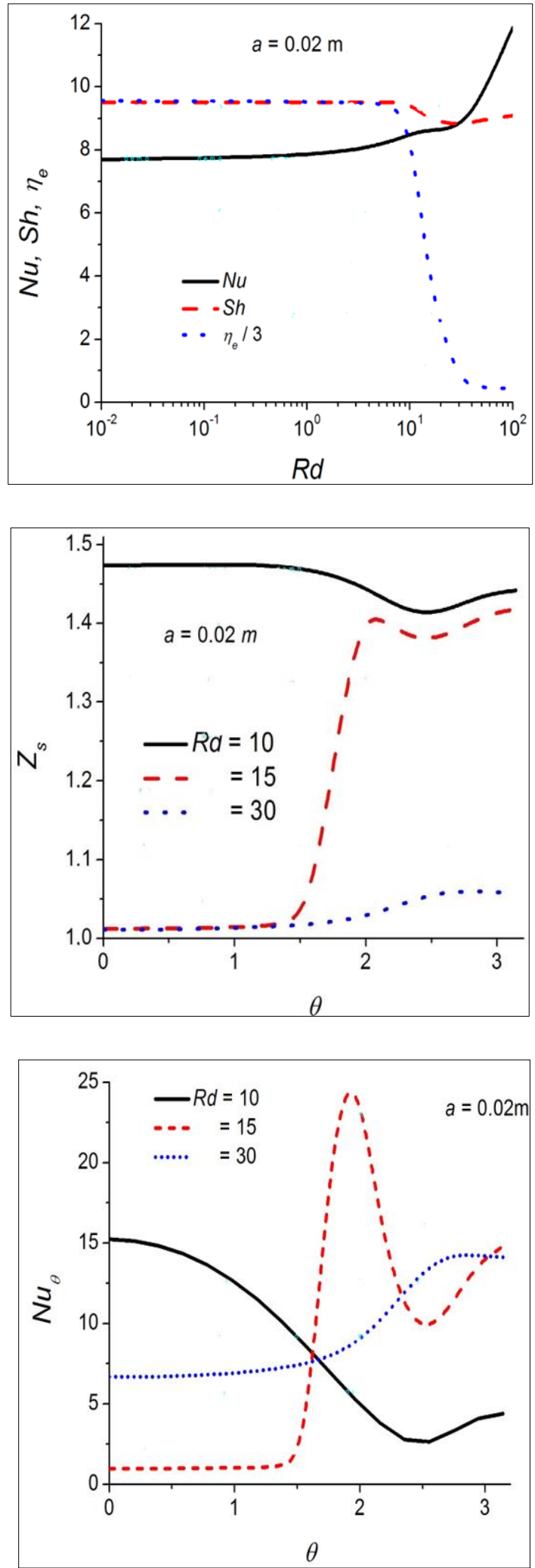

Figure 4. The influence of the radiation conduction parameter $R d$ on $N u$, Sh and $\eta_{e}$ for $\operatorname{Re}=100, B=0.43, \gamma=20.0, \varphi^{2}=0.16$, $K=0.02, \mathfrak{B}=0.18, \mathcal{E}=0.269(a=0.02$ $\mathrm{m}, k_{a}=1 \mathrm{~m}^{-1}, \beta=3 \mathrm{~m}^{-1}$ and $\left.\varepsilon=0.7\right)$

Figure 5. The influence of the radiation conduction parameter $R d$ on the surface dimensionless temperature for $R e=100$, $B=0.43, \gamma=20.0, \varphi^{2}=0.16, K=0.02$, $\mathfrak{B}=0.18$ and $\mathcal{E}=0.269(a=0.02 \mathrm{~m}$, $k_{a}=1 \mathrm{~m}^{-1}, \beta=3 \mathrm{~m}^{-1}$ and $\left.\varepsilon=0.7\right)$
Figure 6. The influence of the radiation conduction parameter $R d$ on the local $N u$ number for $R e=100, B=0.43, \gamma=20.0$, $\varphi^{2}=0.16, K=0.02, \mathfrak{B}=0.18$ and $\mathcal{E}=0.269$ $\left(a=0.02 \mathrm{~m}, k_{a}=1 \mathrm{~m}^{-1}, \beta=3 \mathrm{~m}^{-1}\right.$ and $\left.\varepsilon=0.7\right)$

The influence of thermal radiation on the surface local variables is plotted in Figures 5 - 8 . The three $R d$ values from Figures 5 - 8 describe the following situations (Figures 2 and 3): (1) before the critical domain, $R d=10$; (2) inside the critical domain, $R d=15$; (3) after the critical domain, $R d=30$. Figures 5 - 8 show that for $R d=10$, the variation of $Z, Y, N u_{\theta}$ and $S h_{\theta}$ on the surface of the sphere is the typical variation of the convection - diffusion equations solutions for the flow past a sphere with flow separation. For $R d=15$, one can observe that thermal radiation does not work globally on the surface temperature 
and its effect does not increase gradually with the increase in $R d$. It works locally, with full strength, starting from the front stagnation point. With the increase in $R d$, the effect of thermal radiation extends over to the full surface of the sphere until the rear stagnation point $(R d=30)$. The local maximum values of $N u_{\theta}$ and $S h_{\theta}$ are the result of the following phenomena: (1) due to convection, the cooling of the fluid is faster than the cooling of the sphere surface; (2) cold fluid from the front stagnation point flows and contacts the hot region from the surface of the sphere.
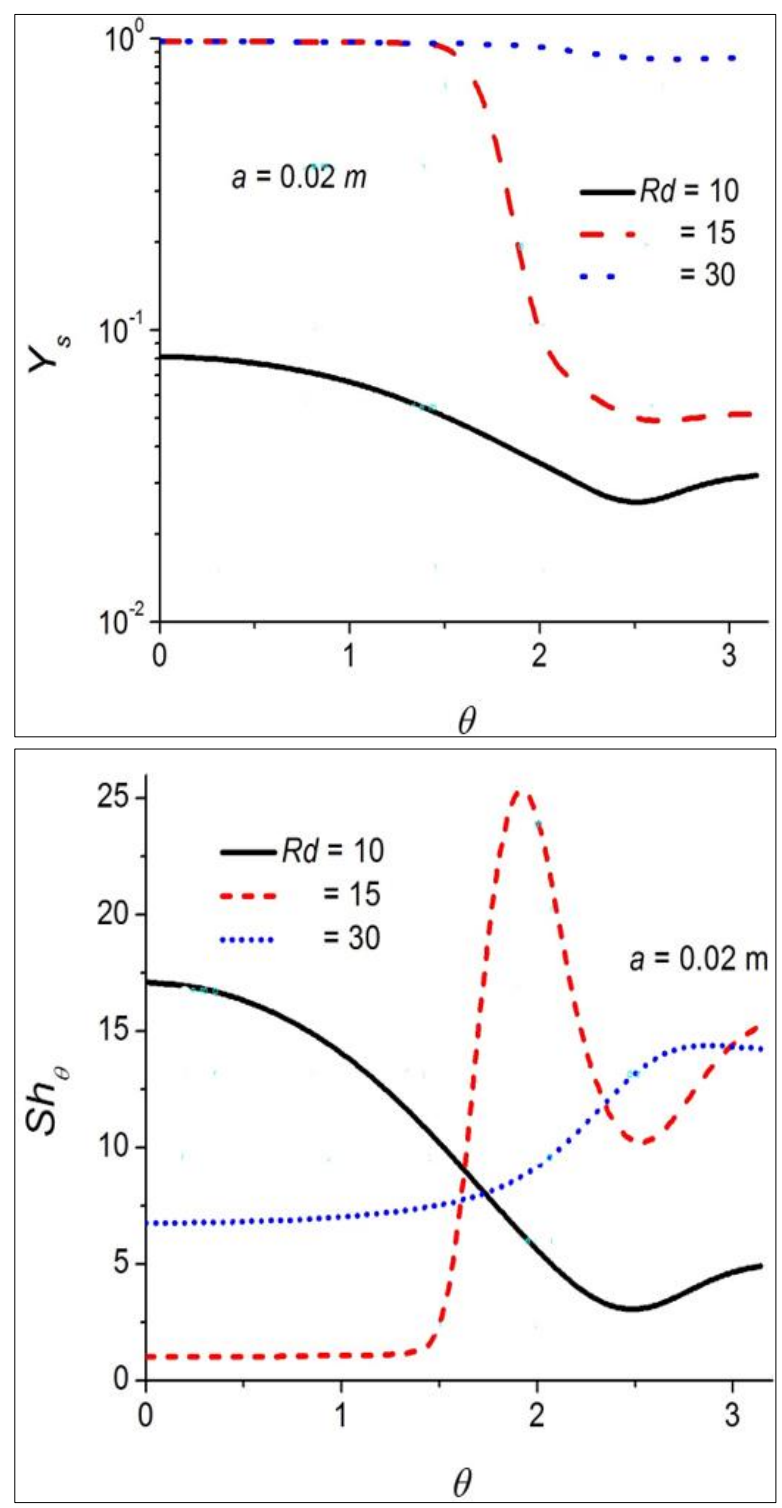

Figure 7. The influence of the radiation conduction parameter $R d$ on the surface dimensionless concentration for $R e=100$, $B=0.43, \gamma=20.0, \varphi^{2}=0.16, K=0.02$
$\mathfrak{B}=0.18$ and $\varepsilon=0.269(a=0.02 \mathrm{~m}$
$k_{a}=1 \mathrm{~m}^{-1}, \beta=3 \mathrm{~m}^{-1}$ and $\left.\varepsilon=0.7\right)$
Figure 8. The influence of the radiation conduction parameter $R d$ on the local $S h$ number for $\operatorname{Re}=100, B=0.43, \gamma=20.0$, $\varphi^{2}=0.16, K=0.02, \mathfrak{B}=0.18$ and $\mathcal{E}=0.269$ $\left(a=0.02 \mathrm{~m}, k_{a}=1 \mathrm{~m}^{-1}, \beta=3 \mathrm{~m}^{-1}\right.$ and $\left.\varepsilon=0.7\right)$

The dimensionless directed - integrated intensity of radiation $G$ is the solution of the diffusion reaction equation (6). Figure 9 shows that the surface profiles of $G$ do not have the usual characteristics of the solution of a diffusion - reaction equation. The coupling between equations (6) and (7) and implicitly the interaction between $Z$ and $G$ determine the shape of the $G$ profiles.

Concerning the effect of the other radiation parameters on the surface catalytic chemical reaction, the numerical experiments made have shown the followings: the increase in the absorption coefficient $k_{a}$ increases the effect of thermal radiation while the increase in the total attenuation factor $\beta$ and the emissivity coefficient $\varepsilon$ decreases the effect of thermal radiation. In the previous sentences, the increase in the effect of thermal radiation means the decrease in the $R d$ value for which the jump occurs. 


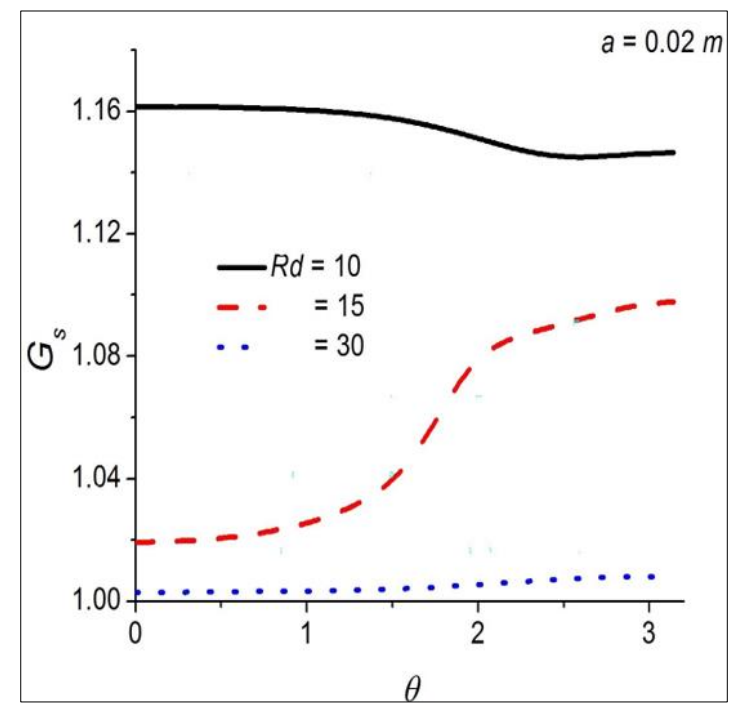

Figure 9. The influence of the radiation conduction parameter $R d$ on the surface dimensionless directed - integrated intensity of radiation for $R e=100$, $B=0.43, \gamma=20.0, \varphi^{2}=0.16, K=0.02$, $+\mathfrak{B}=0.18$ and $\mathcal{E}=0.269(a=0.02 \mathrm{~m}$, $k_{a}=1 \mathrm{~m}^{-1}, \beta=3 \mathrm{~m}^{-1}$ and $\left.\varepsilon=0.7\right)$

\section{Conclusions}

The effect of thermal radiation on the steady-state, heat and transfer from a fluid flow to a sphere with an exothermic catalytic chemical reaction on the surface of the sphere is the topic of the present work. The radiative transfer is modeled by the $P_{1}$ approximation. The case when the conversion of the reactant on the surface of the sphere is almost complete in the absence of thermal radiation is investigated in detail.

The effect of the thermal radiation on the surface catalytic chemical reaction consists of the decrease in the sphere surface temperature. The increase in the radiation - conduction group $R d$ increases the effect of the thermal radiation on the surface catalytic chemical reaction. The variation of the sphere surface average dimensionless temperature and concentration versus $R d$ is similar to a step function. The values of $R d$ for which the jump occurs depend on the radius of the sphere.

The extension of the present analysis to other values of the reaction parameters and convection rates (the products $\operatorname{Re} \operatorname{Pr}$ and $\operatorname{Re} S c$ ) will be a challenge for future works.

\section{List of symbols}

$a$ - radius of the sphere

$B$ - dimensionless adiabatic heat rise (Prater number), $\left(-\Delta H_{R} D C_{\infty}\right) /\left(\lambda T_{\infty}\right)$

$\mathfrak{B}$ - dimensionless group, $\mathfrak{B}=3 \beta a$

$c_{P}$ - heat capacity

$C$ - concentration of reactant

$d$ - diameter of the sphere, $d=2 a$

$D$ - diffusion coefficient

$E$ - activation energy of the chemical reaction

$\mathcal{E}$ - dimensionless group, $\mathcal{E}=\frac{\varepsilon}{2(2-\varepsilon)}$

$G$ - dimensionless directed - integrated intensity of the radiation, $G=\frac{G^{*}}{4 n^{2} \sigma T_{\infty}^{4}}$

$G^{*}$ - dimensional directed - integrated intensity of the radiation

$k_{a}$ - absorption coefficient

$k_{0}$ - pre-exponential factor

$K$ - dimensionless group, $K=k_{a} a$

$n$-index of refraction

$\mathrm{Nu}$ - average Nusselt number defined by relation (12)

$N u_{\theta}-$ local Nusselt number defined by relation (11)

$P r-$ Prandtl number, $P e=\mu / \rho \alpha$ 
$q_{r}$ - dimensionless radiative heat flux vector

$R e-$ sphere Reynolds number, $R e=U_{\infty} d \rho / \mu$

$R d$ - radiation - conduction parameter

$R_{G}-$ gas constant

$r$ - dimensionless radial coordinate, $r^{*} / a$, in spherical coordinate system

$r^{*}$ - radial coordinate in spherical coordinate system

Sc - Schimdt number, $S c=\mu / \rho D$

$S h$ - average Sherwood number defined by relation (12)

$S h_{\theta}-$ local Sherwood number defined by relation (11)

$T$ - temperature

$U_{\infty}$ - velocity far away from the sphere

$V_{R}$ - dimensionless radial velocity component

$V_{\theta}$ - dimensionless tangential velocity component

$Y$-dimensionless concentration of reactant, $C / C_{\infty}$

$Z$-dimensionless temperature, $T / T_{\infty}$

\section{Greek symbols}

$\alpha$ - thermal diffusivity

$\beta$ - extinction coefficient (total attenuation factor)

$\gamma$-dimensionless activation energy (Arrhenius number), $E / R_{G} T_{\infty}$

$\triangle H_{R}$ - heat of reaction

$\varepsilon-$ emissivity coefficient

$\eta_{e}-$ external effectiveness factor defined by relation (10)

$\lambda$ - thermal conductivity

$\mu$ - dynamic viscosity

$\theta$ - polar angle in spherical coordinate system

$\rho$ - density

$\sigma$ - Stefan - Boltzman constant

$\varphi-$ reaction parameter (Thiele modulus), $\varphi=a \sqrt{\frac{\rho k_{0} \exp (-\gamma)}{D}}$

\section{Subscripts}

$\infty$ - refers to a large finite distance from the center of the sphere

$s$ - refers to the surface of the sphere

\section{References}

1. CHUNG, P.M., "Chemically reacting nonequilibrium boundary layers", Advances in Heat Transfer, 2, 1965, p. 109.

2. LINDBERG, R.C., SCHMITZ, R.A., "On the multiplicity of steady states in boundary layer problems with surface reaction”, Chem. Eng. Sci., 24, no. 7, 1969, p. 1113.

3. MIHAIL, R., "A superposition integral equation for catalytic external surfaces", Chem. Eng. Sci., 25, no. 3, 1970, p. 463.

4. MIHAIL, R., "External catalytic reactions in non-Newtonian fluids", Chem. Eng. Sci., 26, no. 12, 1971, p. 2120.

5. MIHAIL, R., TEODORESCU C., "Laminar boundary layer with nonisothermal surface reaction", Chem. Eng. Sci., 30, no. 8, 1975, p. 993.

6. SCHEFER, R.W., "Catalyzed combustion of $\mathrm{H}_{2}$ /Air mixtures in a flat plate boundary layer: II. Numerical model", Combustion Flame 45, 1982, p. 171.

7. A. LINAN, A., TREVINO, C., "Ignition and extinction of catalytic reactions on a flat plate", Combustion Sci. Tech., 38, no. 3-4, 1984, p. 113. 
8. MARKATOU, P., PFEFFERLE L.D., SMOOKE, M.T., "A computational study of methane-air combustion over heated catalytic and non-catalytic surfaces", Combustion Flame, 93, no. 1-2, 1993, p. 185.

9. ELPERIN, T., FOMINYKH, A., "Exact analytical solution of a convective diffusion from a wedge to a flow with a first order chemical reaction at the surface", Int. Comm. Heat Mass Transf., 21, no. 2, 1994, p. 227.

10. ELPERIN, T., FOMINYKH, A., "Mass transfer between a two-dimensional wall jet with a heterogeneous chemical reaction of the first order and a wall: analytical solution", Int. J. Heat Mass Transf., 39, no. 15, 1996, p. 3287.

11. MERKIN, J.H., "A model for isothermal homogeneous-heterogeneous reactions in boundary-layer flow", Math. Comput. Model., 24, no. 8, 1996), p. 125.

12. ELPERIN, T., FOMINYKH, A., ORENBAKH, Z., "Mass transfer with heterogeneous chemical reaction of the first-order in Falkner-Skan flow of a power-law fluid", Chem. Eng. Comm., 192, no. 11, 2005, p. 1505.

13. LINDBERG, R.C., SCHMITZ, R.A., "Multiplicity of steady states with surface reaction on a blunt object in a convective system", Chem. Eng. Sci., 25, no. 5, 1970, p. 901.

14. MIHAIL R., "Shape effects in external surface catalysis", Chem. Eng. Sci., 27, no. 5, 1972, p. 845. 15. GUPALO, Y.P., RYAZANTSEV, Y.S., "Mass and heat transfer from a sphere in laminar flow", Chem. Eng. Sci., 27, no. 1, 1972, p. 61.

16. GUPAlO, Y.P., POLYANIN, A.D., RYAZANTSEV, Y.S., SERGEEV, Y.A., "Convective diffusion to a solid particle in gas flow at non-linear kinetics of the heterogeneous chemical reaction", Dokl. Akad. Nauk. SSSR. 237, 1977, p. 86.

17. POLYANIN, A.D., SERGEEV, Y.A., "Convective diffusion to a reacting in a fluid. Nonlinear surface reaction kinetics”, Int. J. Heat Mass Transf., 23, no. 9, 1980, p. 1171.

18. JUNCU, G., MIHAIL R., "Multigrid solution of the diffusion - convection - reaction equations which describe the mass and/or heat transfer from or to a spherical particle", Comput. Chem. Eng., 13, no. 3, 1989, p. 259.

19. PATERSON, W.R., "Mass transfer to, and reaction on, a sphere immersed in a stationary or flowing gas", Chem. Eng. Sci., 55, no. 17, 2000, pp. 3567.

20. LARSEN, E.W., THÖMMES, G., KLAR, A., SEAÏD, M., GÖTZ, T., "Simplified $P_{\mathrm{N}}$ approximations to the equations of radiative heat transfer and applications", J. Comput. Phys., 183, no. 2, 2002, p. 652.

21. MODEST, M.F., Radiative Heat Transfer, third edition, Academic Press, Oxford, 2013.

22. JUNCU, G., MIHAIL R., "Numerical solution of the steady incompressible Navier-Stokes equations for the flow past a sphere by a multigrid defect correction technique", Int. J. Num. Meth. Fluids, 11, no. 4, 1990, p. 379.

23. WESSELING, P., Principles of Computational Fluid Dynamics, Springer, Berlin, 2001.

24. JOHNSON, T.A., PATEL, V.C., "Flow past a sphere up to a Reynolds number of 300", J. Fluid Mech., 378, 1999, p. 19.

25. WIJNGAARDEN, R.J., KRONBERG, A., WESTERTERP, K.R., Industrial Catalysis: Optimizing Catalysts and Processes, Wiley VCH, Weiheim, 1998.

26. ARIS, R., The Mathematical Theory of Diffusion and Reaction in Permeable Catalysts, Calendron Press, Oxford, 1975.

27. FROMENT, G., BISCHOFF, K.B., DE WILDE, J., Chemical Reactor Analysis and Design, third edition, Wiley, New York, 2011.

Manuscript received: 11.07 .2020 